\title{
Fluctuations of an evaporating black hole from back reaction of its Hawking radiation: Questioning a premise in earlier work
}

\author{
B. L. Hu and Albert Roura \\ Department of Physics, University of Maryland, College Park, Maryland 20742-4111
}

\begin{abstract}
This paper delineates the first steps in a systematic quantitative study of the spacetime fluctuations induced by quantum fields in an evaporating black hole. We explain how the stochastic gravity formalism can be a useful tool for that purpose within a low-energy effective field theory approach to quantum gravity. As an explicit example we apply it to the study of the sphericallysymmetric sector of metric perturbations around an evaporating black hole background geometry. For macroscopic black holes we find that those fluctuations grow and eventually become important when considering sufficiently long periods of time (of the order of the evaporation time), but well before the Planckian regime is reached. In addition, the assumption of a simple correlation between the fluctuations of the energy flux crossing the horizon and far from it, which was made in earlier work on spherically-symmetric induced fluctuations, is carefully analyzed and found to be invalid. Our analysis suggests the existence of an infinite amplitude for the fluctuations of the horizon as a three-dimensional hypersurface. We emphasize the need for understanding and designing operational ways of probing quantum metric fluctuations near the horizon and extracting physically meaningful information.
\end{abstract}

Dedicated to Rafael Sorkin on the occasion of his 60th birthday.

\section{INTRODUCTION}

Studying the dynamics of quantum fields in a fixed curved spacetime, Hawking found that black holes emit thermal radiation with a temperature inversely proportional to their mass [1]. When the back reaction of the quantum fields on the spacetime dynamics is included, one expects that the mass of the black hole decreases as thermal radiation at higher and higher temperatures is emitted. This picture, based on the process known as black hole evaporation, is obtained from semiclassical gravity calculations which are believed to be valid at least before the Planckian scale is reached [2, 3].

Semiclassical gravity [4, 5, 6] is a mean field description that neglects the fluctuations of the spacetime geometry. However, a number of studies have suggested the existence of large fluctuations near black hole horizons [7, 8, 9, 10] (and even instabilities 11]) with characteristic time-scales much shorter than the black hole evaporation time. In all of them ${ }^{1}$ either states which are singular on the horizon (such as the Boulware vacuum for Schwarzschild spacetime) were explicitly considered, or fluctuations were computed with respect to those states and found to be large near the horizon. Whether these huge fluctuations are of a generic nature or an artifact arising from the consideration of states singular on the horizon is an issue that deserves further investigation. On the other hand, the fluctuations for states regular on the horizon were estimated in Ref. 12] and found to be small even when integrated over a time of the order of the evaporation time. These apparently contradictory claims and the fact that most claims on black hole horizon fluctuations were based on qualitative arguments and/or semi-quantitative estimates prompted us to strive for a more quantitative and self-consistent description. (Previous attempts on this problem with similar emphasis by Raval, Sinha and one of us have appeared in Refs. 13, 14]. The apparent difference between the conclusions in Ref. 13] and what is reported here will be explained below.) In contrast to those prior studies, we find here that in those cases the accumulated fluctuations become significant by the time the black hole mass has changed substantially, but well before reaching the Planckian regime. Our result is in agreement with earlier work by Bekenstein 15.

We will study metric fluctuations within a low-energy effective field theory approach to quantum gravity [16], which is commonly believed to provide a valid description for phenomena involving typical length-scales much larger than the Planck length even if the microscopic details of the theory at Planckian scales are not known. This approach has been mainly applied to weak field situations, such as the study of quantum corrections to the Newtonian potential for particles in a Minkowski background [17, 18]. However, it is particularly interesting to apply it also to strong field

\footnotetext{
${ }^{1}$ At least those which provide a relativistic description. The argument in Refs. $7,[8]$ is based on a non-relativistic description and it is not obvious how to make some of our statements precise in that case. However, a natural generalization to the relativistic case is provided in Ref. [10], which does fall into this category.
} 
situations involving cosmological [19] or black hole spacetimes. Here we will consider quantized metric perturbations around a black hole background geometry.

In the spirit of the effective field theory approach, the stochastic gravity formalism 20, 21] provides a more than adequate and perhaps the best available framework to study quantum metric fluctuations because, for one reason at least, the correlation functions that one obtains are equivalent to the quantum correlation functions that would follow from a quantum field theory treatment, up to a given order in an expansion in terms of the inverse number of fields 22, 23. Stochastic gravity allows for a systematic study of the metric fluctuations in a black hole spacetime over and beyond that for the mean value of the background spacetime based on semiclassical gravity (with self-consistent back reaction from the expectation value of the stress tensor). In tackling the problem of metric fluctuations driven by quantum matter field fluctuations for states regular on the horizon (as far as the expectation value of the stress tensor is concerned), the existence of correlations between the outgoing energy flux far from the horizon and a negative energy flux crossing the horizon, based on energy conservation arguments, constitutes an important step in previous investigations [12, 15] (see, however, Refs. [24, 25], where those correlators were shown to vanish in an effectively two-dimensional model). Using semiclassical gravity, such correlations have been confirmed for the expectation value of the energy fluxes, provided that the mass of the black hole is much larger than the Planck mass. However, a more careful analysis, summarized in Sec. IV shows that no such simple connection exists for energy flux fluctuations. It also reveals that the fluctuations on the horizon, as a three-dimensional hypersurface, are in fact divergent and one needs to find an adequate way of probing the metric fluctuations near the horizon and extracting physically meaningful information. The non-existence of this commonly invoked relation in this whole subject matter illustrates the limitations of heuristic arguments and the necessity of a detailed and consistent formalism to study the fluctuations near the horizon.

We close this introduction with a few technical remarks. First, we will restrict our attention to the sphericallysymmetric sector of metric fluctuations, which necessarily implies a partial description of the fluctuations. Note, however, that contrary to the case for semiclassical gravity solutions, even if one starts with spherically-symmetric initial conditions, the stress tensor fluctuations will induce fluctuations involving higher multipoles. Thus, the multipole structure of the fluctuations is far richer than that of spherically-symmetric semiclassical gravity solutions, but this also means that obtaining a complete solution (including all multipoles) for fluctuations rather than the mean value, even a particular one, is much more difficult.

Second, for black hole masses much larger than the Planck mass (otherwise the effective field theory description will break down anyway), one can introduce a useful adiabatic approximation involving inverse powers of the black hole mass. To obtain results to lowest order, it is sufficient to compute the expectation value of the stress tensor operator and its correlation functions in Schwarzschild spacetime. The corrections proportional to higher powers of the inverse mass can be neglected for sufficiently massive black holes.

Third, when studying the dynamics of induced metric fluctuations, the additional contribution to the stress tensor expectation value which results from evaluating it using the perturbed metric is often neglected. In the consideration of fluctuations for an evaporating black hole such a term (which will be denoted by $\left\langle\hat{T}_{a b}^{(1)}[g+h]\right\rangle_{\text {ren }}$ in Sec. IIII) becomes important when it builds up for long times. The importance of this term is clear when comparing with the simple estimate made by $\mathrm{Wu}$ and Ford in Ref. [12], where $\left\langle\hat{T}_{a b}^{(1)}[g+h]\right\rangle_{\text {ren }}$ was neglected and the fluctuations were found to be small even when integrated over long times, of the order of the evaporation time of the black hole.

The paper is organized as follows. In Sec. Пwe briefly review the results for the mean evolution of an evaporating black hole obtained in the context of semiclassical gravity. The stochastic gravity formalism is then applied in Sec. III to the study of the spherically-symmetric sector of fluctuations around the semiclassical gravity solution for an evaporating black hole. The existence of an exact correlation between the fluctuations of the negative energy flux crossing the horizon and the flux far from it has been previously assumed. In this paper we want to question this assumption, but in the presentation in Sec. III] we make such an assumption to be in line with the literature. In Sec. IV we present a careful analysis of this assumption, and show that it is invalid. Further details can be found in a longer paper on the same subject [26]. Finally, in Sec. $\nabla$ we discuss several implications of our results and suggest some directions for further investigation.

Throughout the paper we use Planckian units with $\hbar=c=G=1$ and the $(+,+,+)$ convention of Ref. 27]. We also make use of the abstract index notation of Ref. [28]. Latin indices denote abstract indices, whereas Greek indices are employed whenever a particular coordinate system is considered.

\section{MEAN EVOLUTION OF AN EVAPORATING BLACK HOLE}

Semiclassical gravity provides a mean field description of gravitational back-reaction problems in which the quantum effects of the matter fields can be important [4, 5, 6]. It is believed to be applicable to situations involving length-scales much larger than the Planck scale and for which the quantum back-reaction effects due to the metric itself can be 
neglected as compared to those due to the matter fields. In semiclassical gravity the spacetime geometry is described by a classical metric while the matter fields are quantized. The dynamics of the metric is governed by the semiclassical Einstein equation:

$$
G_{a b}[g]=\kappa\left\langle\hat{T}_{a b}[g]\right\rangle_{\text {ren }},
$$

where $\left\langle\hat{T}_{a b}[g]\right\rangle_{\text {ren }}$ is the renormalized expectation value of the stress tensor operator of the quantum matter fields and $\kappa=8 \pi / m_{\mathrm{p}}^{2}$ with $m_{\mathrm{p}}^{2}$ being the Planck mass. Both the semiclassical Einstein equation and the equation of motion for the matter fields evolving in that geometry, whose solution is needed to evaluate $\left\langle\hat{T}_{a b}[g]\right\rangle_{\text {ren }}$, must be solved self-consistently.

An important application of semiclassical gravity is the study of black hole evaporation due to the back reaction of the Hawking radiation emitted by the black hole on the spacetime geometry. This has been studied in some detail for spherically symmetric black holes 2, 3]. For a general spherically-symmetric metric there always exists a system of coordinates in which it takes the form

$$
d s^{2}=-e^{2 \psi(v, r)}(1-2 m(v, r) / r) d v^{2}+2 e^{\psi(v, r)} d v d r+r^{2}\left(d \theta^{2}+\sin ^{2} \theta d \varphi^{2}\right) .
$$

This completely fixes the gauge freedom under local diffeomorphism transformations except for an arbitrary function of $v$ that can be added to the function $\psi(v, r)$ and is related to the freedom in reparametrizing $v$ (we will see below how this can also be fixed). In general this metric exhibits an apparent horizon, where the expansion of the outgoing radial null geodesics vanishes and which separates regions with positive and negative expansion for those geodesics, at those radii that correspond to (odd degree) zeroes of the $v v$ metric component. Throughout the paper the location of the apparent horizon will be denoted by $r_{\mathrm{AH}}(v)=2 M(v)$, where $M(v)$ satisfies the equation $2 m(2 M(v), v)=2 M(v)$.

Spherical symmetry implies that the components $T_{\theta r}, T_{\theta v}, T_{\varphi r}$ and $T_{\varphi v}$ vanish and the remaining components are independent of the angular coordinates. Under these conditions the various components of the semiclassical Einstein equation associated with the metric in Eq. (2) become

$$
\begin{aligned}
& \frac{\partial m}{\partial v}=4 \pi r^{2} T_{v}^{r}, \\
& \frac{\partial m}{\partial r}=-4 \pi r^{2} T_{v}^{v}, \\
& \frac{\partial \psi}{\partial r}=4 \pi r T_{r r},
\end{aligned}
$$

where from now on we will simply use $T_{\mu \nu}$ to denote the expectation value $\left\langle\hat{T}_{\mu \nu}[g]\right\rangle_{\text {ren }}$ and employ Planckian units (with $m_{\mathrm{p}}^{2}=1$ ). Note that the arbitrariness in $\psi$ can be eliminated by choosing a parametrization of $v$ such that $\psi$ takes a particular value at a given radius (we will choose that it vanishes at $r=2 M(v)$, where the apparent horizon is located); $\psi$ is then entirely fixed by Eq. (15).

Solving Eqs. (3)-(5) is no easy task. However, one can introduce a useful adiabatic approximation in the regime where the mass of the black hole is much larger than the Planck mass, which is in any case a necessary condition for the semiclassical treatment to be valid. What this entails is that when $M \gg 1$ (remember that we are using Planckian units) for each value of $v$ one can simply substitute $T_{\mu \nu}$ by its "parametric value" - by this we mean the expectation value of the stress energy tensor of the quantum field in a Schwarzschild black hole with a mass corresponding to $M(v)$ evaluated at that value of $v$. This is in contrast to its dynamical value, which should be determined by solving self-consistently the semiclassical Einstein equation for the spacetime metric and the equations of motion for the quantum matter fields. This kind of approximation introduces errors of higher order in $L_{\mathrm{H}} \equiv B / M^{2}$ ( $B$ is a dimensionless parameter that depends on the number of massless fields and their spins and accounts for their corresponding grey-body factors; it has been estimated to be of order $10^{-4}[29]$ ), which are very small for black holes well above Planckian scales. These errors are due to the fact that $M(v)$ is not constant and that, even for a constant $M(v)$, the resulting static geometry is not exactly Schwarzschild because the vacuum polarization of the quantum fields gives rise to a non-vanishing $\left.\left\langle\hat{T}_{a b}[g]\right\rangle_{\text {ren }} 30\right]$.

The expectation value of the stress tensor for Schwarzschild spacetime has been found to correspond to a thermal flux of radiation (with $T_{v}^{r}=L_{\mathrm{H}} /\left(4 \pi r^{2}\right)$ ) for large radii and of order $L_{\mathrm{H}}$ near the horizon ${ }^{2}$ [31, 32, 33, 34, 35]. This

\footnotetext{
2 The natural quantum state for a black hole formed by gravitational collapse is the Unruh vacuum, which corresponds to the absence of incoming radiation far from the horizon. The expectation value of the stress tensor operator for that state is finite on the future horizon of Schwarzschild, which is the relevant one when identifying a region of the Schwarzschild geometry with the spacetime outside the collapsing matter for a black hole formed by gravitational collapse.
} 
shows the consistency of the adiabatic approximation for $L_{\mathrm{H}} \ll 1$ : the right-hand side of Eqs. (31)-(15) contains terms of order $L_{\mathrm{H}}$ and higher, so that the derivatives of $m(v, r)$ and $\psi(v, r)$ are indeed small. Furthermore, one can use the $v$ component of the stress-energy conservation equation (where terms of order $L_{\mathrm{H}}^{2}$ and higher are neglected here)

$$
\frac{\partial\left(r^{2} T_{v}^{r}\right)}{\partial r}+r^{2} \frac{\partial T_{v}^{v}}{\partial v}=0
$$

to relate the $T_{v}^{r}$ components on the horizon and far from it. Integrating Eq. (6) radially, one gets

$$
\left(r^{2} T_{v}^{r}\right)(r=2 M(v), v)=\left(r^{2} T_{v}^{r}\right)(r \approx 6 M(v), v)+O\left(L_{\mathrm{H}}^{2}\right),
$$

where we considered a radius sufficiently far from the horizon, but not arbitrarily far (i.e. $2 M(v) \ll r \ll M(v) / L_{\mathrm{H}}$ ). The second condition is necessary to ensure that the size of the horizon has not changed much since the value of $v^{\prime}$ at which the radiation crossing the sphere of radius $r$ at time $v$ left the region close to the horizon. Note that while in the nearly flat region (for large radii) $T_{v}^{r}$ corresponds to minus the outgoing energy flux crossing the sphere of radius $r$, on the horizon, where $d s^{2}=2 e^{\psi(v, r)} d v d r+r^{2}\left(d \theta^{2}+\sin ^{2} \theta d \varphi^{2}\right), T_{v}^{r}$ equals $T_{v v}$, which corresponds to the null energy flux crossing the horizon. Hence, Eq. (77) relates the positive energy flux radiated away far from the horizon and the negative energy flux crossing the horizon. Taking into account this connection between energy fluxes and evaluating Eq. (3) on the apparent horizon, we finally get the equation governing the evolution of its size:

$$
\frac{d M}{d v}=-\frac{B}{M^{2}}
$$

Unless $M(v)$ is constant, the event horizon and the apparent horizon do not coincide. However, in the adiabatic regime their radii are related, differing by a quantity of higher order in $L_{\mathrm{H}}: r_{\mathrm{EH}}(v)=r_{\mathrm{AH}}(v)\left(1+O\left(L_{\mathrm{H}}\right)\right)$.

\section{SPHERICALLY-SYMMETRIC INDUCED FLUCTUATIONS}

There are situations in which the fluctuations of the stress tensor operator and the metric fluctuations that they induce may be important, so that the mean field description provided by semiclassical gravity is incomplete and even fails to capture the most relevant phenomena (the generation of primordial cosmological perturbations constitutes a clear example of that). The stochastic gravity formalism [20, 21] provides a framework to study those fluctuations. Its centerpiece is the Einstein-Langevin equation

$$
G_{a b}^{(1)}[g+h]=\kappa\left\langle\hat{T}_{a b}^{(1)}[g+h]\right\rangle_{\mathrm{ren}}+\kappa \xi_{a b}[g],
$$

which governs the dynamics of the metric fluctuations around a background metric $g_{a b}$ that corresponds to a given solution of semiclassical gravity. The superindex (1) indicates that only the terms linear in the metric perturbations should be considered, and $\xi_{a b}$ is a Gaussian stochastic source with vanishing expectation value and correlation function ${ }^{3}$ $\left\langle\xi_{a b}(x) \xi_{c d}\left(x^{\prime}\right)\right\rangle_{\xi}=(1 / 2)\left\langle\left\{\hat{t}_{a b}(x), \hat{t}_{c d}\left(x^{\prime}\right)\right\}\right\rangle$ (with $\left.\hat{t}_{a b} \equiv \hat{T}_{a b}-\left\langle\hat{T}_{a b}\right\rangle\right)$, where the term on the right-hand side, which accounts for the stress tensor fluctuations within this Gaussian approximation, is commonly known as the noise kernel and denoted by $N_{a b c d}\left(x, x^{\prime}\right)$. In this framework the metric perturbations are still classical but stochastic. Nevertheless, one can show that the correlation functions for the metric perturbations that one obtains in stochastic gravity are equivalent through order $1 / N$ to the quantum correlation functions that would follow from a quantum field theory treatment when considering a large number of fields $N$ [22, 23]. In particular, the symmetrized two-point function consists of two contributions: intrinsic and induced fluctuations. The intrinsic fluctuations are a consequence of the quantum width of the initial state of the metric perturbations, and they are obtained in stochastic gravity by averaging over the initial conditions for the solutions of the homogeneous part of Eq. (9) distributed according to the reduced Wigner function associated with the initial quantum state of the metric perturbations. On the other hand, the induced fluctuations are due to the quantum fluctuations of the matter fields interacting with the metric perturbations, and they are obtained by solving the Einstein-Langevin equation using a retarded propagator with vanishing initial conditions.

In this section, we will apply the stochastic gravity formalism to the study of the spherically-symmetric sector (i.e., the monopole contribution, which corresponds to $l=0$, in a multipole expansion in terms of spherical harmonics

\footnotetext{
3 Throughout the paper we use the notation $\langle\ldots\rangle_{\xi}$ for stochastic averages over all possible realizations of the source $\xi_{a b}$ to distinguish them from quantum averages, which are denoted by $\langle\ldots\rangle$.
} 
$\left.Y_{l m}(\theta, \phi)\right)$ of metric fluctuations for an evaporating black hole. In this case only induced fluctuations are possible. The fact that intrinsic fluctuations cannot exist can be clearly seen if one neglects vacuum polarization effects, since Birkhoff's theorem forbids the existence of spherically-symmetric free metric perturbations outside the vacuum region of a spherically-symmetric black hole that keep the ADM mass constant. Even when vacuum polarization effects are included, spherically-symmetric perturbations, characterized by $m(v, r)$ and $\psi(v, r)$, are not independent degrees of freedom. This follows from Eqs. (3)-(5), which can be regarded as constraint equations.

The fluctuations of the stress tensor are inhomogeneous and non-spherically-symmetric even if we choose a spherically-symmetric vacuum state for the matter fields (spherical symmetry simply implies that the angular dependence of the noise kernel in spherical coordinates is entirely given by the relative angle between the spacetime points $x$ and $y$ ). This means that, in contrast to the semiclassical gravity case, projecting onto the $l=0$ sector of metric perturbations does not give an exact solution of the Einstein-Langevin equation in the stochastic gravity approach that we have adopted here. Nevertheless, restricting to spherical symmetry in this way gives more accurate results than two-dimensional dilaton-gravity models resulting from simple dimensional reduction [36, 37, 38]. This is because we project the solutions of the Einstein-Langevin equation just at the end, rather than considering only the contribution of the $s$-wave modes to the classical action for both the metric and the matter fields from the very beginning. Hence, an infinite number of modes for the matter fields with $l \neq 0$ contribute to the $l=0$ projection of the noise kernel, whereas only the $s$-wave modes for each matter field would contribute to the noise kernel if dimensional reduction had been imposed right from the start, as done in Refs. 24, 25, 39] as well as in studies of two-dimensional dilaton-gravity models.

The Einstein-Langevin equation for the spherically-symmetric sector of metric perturbations can be obtained by considering linear perturbations of $m(v, r)$ and $\psi(v, r)$, projecting the stochastic source that accounts for the stress tensor fluctuations to the $l=0$ sector, and adding it to the right-hand side of Eqs. (3)-(5). We will focus our attention on the equation for the evolution of $\eta(v, r)$, the perturbation of $m(v, r)$ :

$$
\frac{\partial(m+\eta)}{\partial v}=-\frac{B}{(m+\eta)^{2}}+4 \pi r^{2} \xi_{v}^{r}+O\left(L_{\mathrm{H}}^{2}\right)
$$

which reduces, after neglecting terms of order $L_{\mathrm{H}}^{2}$ or higher, to the following equation to linear order in $\eta$ :

$$
\frac{\partial \eta}{\partial v}=\frac{2 B}{m^{3}} \eta+4 \pi r^{2} \xi_{v}^{r}
$$

It is important to emphasize that in Eq. (10) we assumed that the change in time of $\eta(v, r)$ is sufficiently slow so that the adiabatic approximation employed in the previous section to obtain the mean evolution of $m(v, r)$ can also be applied to the perturbed quantity $m(v, r)+\eta(v, r)$. This is guaranteed as long as the term corresponding to the stochastic source is of order $L_{\mathrm{H}}$ or higher, a point that will be discussed below.

Obtaining the noise kernel which determines the correlation function for the stochastic source is highly nontrivial even if we compute it on the Schwarzschild spacetime, which is justified in the adiabatic regime for the background geometry. As implicitly done in prior work (for instance in Refs. 12, 15]; see, however, Refs. 24, 25]), we will assume in this section that the fluctuations of the radiated energy flux far from the horizon are exactly correlated with the fluctuations of the negative energy flux crossing the horizon. This is a crucial assumption which implies an enormous simplification and allows a direct comparison with the results in the existing literature, and its validity will be analyzed more carefully in the next section. ${ }^{4}$

Since the generation of Hawking radiation is especially sensitive to what happens near the horizon, from now on we will concentrate on the metric perturbations near the horizon ${ }^{5}$ and consider $\eta(v)=\eta(v, 2 M(v))$. Assuming that the fluctuations of the energy flux crossing the horizon and those far from it are exactly correlated, from Eq. (11) we have

$$
\frac{d \eta(v)}{d v}=\frac{2 B}{M^{3}(v)} \eta(v)+\xi(v)
$$

\footnotetext{
4 This simple relation between the energy flux crossing the horizon and the flux far from it is valid for the expectation value of the stress tensor, which is based on an energy conservation argument for the $T_{v}^{r}$ component. In most of the literature this relation is assumed to hold also for fluctuations. However, in the next section we will show that this is an incorrect assumption. Therefore, results derived from this assumption and conclusions drawn are in principle suspect. (This misstep is understandable because most authors have not acquired as much insight into the nature of fluctuations phenomena as now.) Our investigation testifies to the necessity of a complete reexamination of all cases afresh. In fact, as we will show in the longer paper following, an evaluation of the noise kernel near the horizon seems unavoidable for the consideration of fluctuations and back-reaction issues.

5 This means that possible effects on the Hawking radiation due to the fluctuations of the potential barrier for the radial mode functions will be missed by our analysis.
} 
where $\xi(v) \equiv\left(4 \pi r^{2} \xi_{v}^{r}\right)(v, r \approx 6 M(v))$. The correlation function for the spherically-symmetric fluctuation $\xi(v)$ is determined by the integral over the whole solid angle of the $N_{v v}^{r}$ component of the noise kernel, which is given by $(1 / 2)\left\langle\left\{\hat{t}_{v}^{r}(x), \hat{t}_{v}^{r}\left(x^{\prime}\right)\right\}\right\rangle$. The $l=0$ fluctuations of the energy flux of Hawking radiation, characterized by $(1 / 2)\left\langle\left\{\hat{t}_{v}^{r}(x), \hat{t}_{v}^{r}\left(x^{\prime}\right)\right\}\right\rangle$, far from a black hole formed by gravitational collapse have been studied in Ref. 12]. Its main features are a correlation time of order $M$ and a characteristic fluctuation amplitude of order $\epsilon_{0} / M^{4}$ (this is the result of smearing the stress tensor two-point function, which diverges in the coincidence limit, over a period of time of the order of the correlation time). The order of magnitude of $\epsilon_{0}$ has been estimated to lie between $0.1 B$ and $B$ [12, 15]. For simplicity, we will consider quantities smeared over a time of order $M$. We can then introduce the Markovian approximation $\left(\epsilon_{0} / M^{3}(v)\right) \delta\left(v-v^{\prime}\right)$, which coarse-grains the information on features corresponding to time-scales shorter than the correlation time $M$. Under those conditions $r^{2} \xi_{v}^{r}$ is of order $1 / M^{2}$ and the adiabatic approximation made when deriving Eq. (10) is justified.

The stochastic equation (12) can be solved in the usual way and the correlation function for $\eta(v)$ can then be computed. Alternatively, one can follow Bekenstein [15] and derive directly an equation for $\left\langle\eta^{2}(v)\right\rangle_{\xi}$. This is easily done multiplying Eq. (12) by $\eta(v)$ and taking the expectation value. The result is

$$
\frac{d}{d v}\left\langle\eta^{2}(v)\right\rangle_{\xi}=\frac{4 B}{M^{3}(v)}\left\langle\eta^{2}(v)\right\rangle_{\xi}+2\langle\eta(v) \xi(v)\rangle_{\xi}
$$

For delta-correlated noise (the Stratonovich prescription is the appropriate one in this case), $\langle\eta(v) \xi(v)\rangle_{\xi}$ equals one half the time-dependent coefficient multiplying the delta function $\delta\left(v-v^{\prime}\right)$ in the expression for $\left\langle\xi(v) \xi\left(v^{\prime}\right)\right\rangle_{\xi}$, which is given by $\epsilon_{0} / M^{3}(v)$ in our case. Taking that into account, Eq. (13) becomes

$$
\frac{d}{d v}\left\langle\eta^{2}(v)\right\rangle_{\xi}=\frac{4 B}{M^{3}(v)}\left\langle\eta^{2}(v)\right\rangle_{\xi}+\frac{\epsilon_{0}}{M^{3}(v)} .
$$

Finally, it is convenient to change from the $v$ coordinate to the mass function $M(v)$ for the background solution. Eq. (14) can then be rewritten as

$$
\frac{d}{d M}\left\langle\eta^{2}(M)\right\rangle_{\xi}=-\frac{4}{M}\left\langle\eta^{2}(M)\right\rangle_{\xi}-\frac{\left(\epsilon_{0} / B\right)}{M} .
$$

The solutions of this equation are given by

$$
\left\langle\eta^{2}(M)\right\rangle_{\xi}=\left\langle\eta^{2}\left(M_{0}\right)\right\rangle_{\xi}\left(\frac{M_{0}}{M}\right)^{4}+\frac{\epsilon_{0}}{4 B}\left[\left(\frac{M_{0}}{M}\right)^{4}-1\right] .
$$

Provided that the fluctuations at the initial time corresponding to $M=M_{0}$ are negligible (much smaller than $\sqrt{\epsilon_{0} / 4 B} \sim 1$ ), the fluctuations become comparable to the background solution when $M \sim M_{0}^{2 / 3}$. Note that fluctuations of the horizon radius of order one in Planckian units do not correspond to Planck scale physics because near the horizon $\Delta R=r-2 M$ corresponds to a physical distance $L \sim \sqrt{M \Delta R}$, as can be obtained from the line element for Schwarzschild, $d s^{2}=-(1-2 M / r) d t^{2}+(1-2 M / r)^{-1} d r^{2}+r^{2}\left(d \theta^{2}+\sin ^{2} \theta d \varphi^{2}\right)$, by considering pairs of points at constant $t$. So $\Delta R \sim 1$ corresponds to $L \sim \sqrt{M}$, whereas a physical distance of order one is associated with $\Delta R \sim 1 / M$, which corresponds to an area change of order one for spheres with those radii. One can, therefore, have initial fluctuations of the horizon radius of order one for physical distances well above the Planck length provided that we consider a black hole with a mass much larger than the Planck mass. One expects that the fluctuations for states that are regular on the horizon correspond to physical distances not much larger than the Planck length, so that the horizon radius fluctuations would be much smaller than one for sufficiently large black hole masses. Nevertheless, that may not be the case when dealing with states which are singular on the horizon, with estimated fluctuations of order $M^{1 / 3}$ or even $\sqrt{M}[9,10,11]$. Confirming that the fluctuations are indeed so small for regular states and verifying how generic, natural and stable they are as compared to singular ones is a topic that we plan to address in future investigations.

Our result for the growth of the fluctuations of the size of the black hole horizon agrees with the result obtained by Bekenstein in Ref. [15] and implies that, for a sufficiently massive black hole (with a few solar masses or a supermassive black hole), the fluctuations become important before the Planckian regime is reached. Strictly speaking, one cannot expect that a linear treatment of the perturbations provides an accurate result when the fluctuations become comparable to the mean value, but it signals a significant growth of the fluctuations (at least until the nonlinear effects on the perturbation dynamics become relevant).

This growth of the fluctuations which was found by Bekenstein and confirmed here via the Einstein-Langevin equation seems to be in conflict with the estimate given by Wu and Ford in Ref. [12]. According to their estimate, 
the accumulated mass fluctuations over a period of the order of the black hole evaporation time $\left(\Delta t \sim M_{0}^{3}\right)$ would be of the order of the Planck mass. The discrepancy is due to the fact that the first term on the right-hand side of Eq. (12), which corresponds to the perturbed expectation value $\left\langle\hat{T}_{a b}^{(1)}[g+h]\right\rangle_{\text {ren }}$ in Eq. (9), was not taken into account in Ref. 12]. The larger growth obtained here is a consequence of the secular effect of that term, which builds up in time (slowly at first, during most of the evaporation time, and becoming more significant at late times when the mass has changed substantially) and reflects the unstable nature of the background solution for an evaporating black hole. ${ }^{6}$

All this can be qualitatively understood as follows. Consider an evaporating black hole with initial mass $M_{0}$ and suppose that the initial mass is perturbed by an amount $\delta M_{0}=1$. The mean evolution for the perturbed black hole (without taking into account any fluctuations) leads to a mass perturbation that grows like $\delta M=\left(M_{0} / M\right)^{2} \delta M_{0}=$ $\left(M_{0} / M\right)^{2}$, so that it becomes comparable to the unperturbed mass $M$ when $M \sim M_{0}^{2 / 3}$, which coincides with the result obtained above. Such a coincidence has a simple explanation: the fluctuations of the Hawking flux slowly accumulated during most of the evaporating time, which are of the order of the Planck mass, as found by Wu and Ford, give a dispersion of that order for the mass distribution at the time when unstable nature of the small perturbations around the background solution start to become significant.

\section{CORRELATION BETWEEN OUTGOING AND INGOING ENERGY FLUXES}

In this section we will analyze more carefully whether the simple relation between the energy flux crossing the horizon and the flux far from it also holds for the fluctuations. One can find simple arguments which show that those correlations vanish in two-dimensional spacetimes [26. Indeed, the correlation function for the outgoing and ingoing null energy fluxes in an effectively two-dimensional model was explicitly computed in Refs. 24, 25] and found to vanish. On the other hand, in four dimensions the correlation function does not vanish in general and correlations between outgoing and ingoing fluxes do exist near the horizon (at least partially). We plan to elaborate further on these points in Ref. [26].

For black hole masses much larger than the Planck mass, one can use the adiabatic approximation for the background mean evolution. Therefore, to lowest order in $L_{\mathrm{H}}$ one can compute the fluctuations of the stress tensor in Schwarzschild spacetime. In Schwarzschild, the amplitude of the fluctuations of $r^{2} \hat{T}_{v}^{r}$ far from the horizon is of order $1 / M^{2}(=$ $M^{2} / M^{4}$ ) when smearing over a correlation time of order $M$, which one can estimate for a hot thermal plasma in flat space [40, 41] (see Ref. [12] for a more accurate computation of the fluctuations of $r^{2} \hat{T}_{v}^{r}$ far from the horizon). The amplitude of the fluctuations of $r^{2} \hat{T}_{v}^{r}$ is thus of the same order as its expectation value. However, their derivatives with respect to $v$ are rather different: since the characteristic variation times for the expectation value and the fluctuations are $M^{3}$ and $M$ respectively, $\partial\left(r^{2} T_{v}^{r}\right) / \partial v$ is of order $1 / M^{5}$ whereas $\partial\left(r^{2} \xi_{v}^{r}\right) / \partial v$ is of order $1 / M^{3}$. This implies an additional contribution of order $L_{\mathrm{H}}$ due to the second term in Eq. (6) if one radially integrates the same equation applied to stress tensor fluctuations (the stochastic source in the Einstein-Langevin equation). Hence, in contrast to the case of the mean value, the contribution from the second term in Eq. (6) cannot be neglected when radially integrating since it is of the same order as the contributions from the first term, and one can no longer obtain a simple relation between the outgoing energy flux far from the horizon and the energy flux crossing the horizon.

So far we have argued that the method employed for the mean value cannot be employed for the fluctuations. Although one expects that $r^{2} \xi_{v}^{r}$ on the horizon and far from it will not be equal when including the contributions that results from radially integrating the second term in Eq. (6), one might wonder whether there is a possibility that those contributions would somehow cancel out. That possibility can, however, be excluded using the following argument. The smeared correlation function

$$
\int d v h(v) \int d v^{\prime} h\left(v^{\prime}\right) r^{4}\left\langle\xi_{v}^{r}(v, r) \xi_{v}^{r}\left(v^{\prime}, r\right)\right\rangle_{\xi},
$$

where $h(v)$ is some appropriate smearing function and $\xi_{v}^{r}(v, r)$ has already been integrated over the whole solid angle, is divergent on the horizon but finite far from it. Therefore, $r^{2} \xi_{v}^{r}$ on the horizon and far from it cannot be equal for each value of $v$.

\footnotetext{
${ }^{6}$ A clarification between our results and the claims by Hu, Raval and Sinha in Ref. [13] is in place here: both use the stochastic gravity framework and perform an analysis based on the Einstein-Langevin equation, so there should be no discrepancy. However, the claim in Ref. 13] was based on a qualitative argument that focused on the stochastic source, but missed the fact that the perturbations around the mean are unstable for an evaporating black hole. Once this is taken into account, agreement with the result obtained here is recovered.
} 
Let us discuss in some more detail the fact that certain smearings of the quantity $r^{4}\left\langle\xi_{v}^{r}(v, r) \xi_{v}^{r}\left(v^{\prime}, r\right)\right\rangle_{\xi}$ are divergent on the horizon but finite far from it. The smeared correlation function is related to the noise kernel as follows:

$$
\int d v d v^{\prime} h(v) h\left(v^{\prime}\right) r^{4}\left\langle\xi_{v}^{r}(v, r) \xi_{v}^{r}\left(v^{\prime}, r\right)\right\rangle_{\xi}=r^{4} \int d v d v^{\prime} h(v) h\left(v^{\prime}\right) \int d \Omega d \Omega^{\prime} N_{v v}^{r r}\left(v, r, \theta, \varphi ; v^{\prime}, r, \theta^{\prime}, \varphi^{\prime}\right) .
$$

The noise kernel is divergent in the coincident limit or for null-separated points. Smearing the noise kernel along all directions gives a finite result. However, although certain partial smearings also give a finite result, others do not. For instance, smearing along a timelike direction yields a finite result, whereas smearing on a spacelike hypersurface yields in general a divergent result 42]. On the other hand, the result of smearing along two "transverse" null directions (two null directions sharing the same orthogonal spacelike 2-surfaces) is also finite, but not for a smearing along just one null direction even if we also smear along the orthogonal spacelike directions. For $r>2 M$ Eq. (18) corresponds to a smearing along a timelike direction and gives a finite result for the smeared correlation function, but on the horizon it corresponds to a smearing along a single null direction and it is divergent.

The proof of the results described in the previous paragraph will be provided in Ref. [26] by considering a smearing along all directions and then taking the limit in which the smearing size along one of the null directions vanishes. It proceeds in two steps. First, it is shown in the flat space case. Then it is generalized to curved spacetimes using a quasilocal expansion in terms of Riemann normal coordinates.

\section{DISCUSSION}

Using the stochastic gravity formalism, in Sec. [II] we found that the spherically-symmetric fluctuations of the horizon size of an evaporating black hole become important at late times, and even comparable to its mean value when $M \sim M_{0}^{2 / 3}$, where $M_{0}$ is the mass of the black hole at some initial time when the fluctuations of the horizon radius are much smaller than the Planck length. ${ }^{7}$ This is consistent with the result previously obtained by Bekenstein in Ref. [15].

It is important to realize that for a sufficiently massive black hole, the fluctuations become significant well before the Planckian regime is reached. More specifically, for a solar mass black hole they become comparable to the mean value when the black hole radius is of the order of $10 \mathrm{~nm}$, whereas for a supermassive black hole with $M \sim 10^{7} M_{\odot}$, that happens when the radius reaches a size of the order of $1 \mathrm{~mm}$. One expects that in those circumstances the low-energy effective field theory approach of stochastic gravity should provide a reliable description.

Due to the nonlinear nature of the back-reaction equations, such as Eq. (10), the fact that the fluctuations can grow and become comparable to the mean value implies non-negligible corrections to the dynamics of the mean value itself. This can be seen by expanding Eq. (10) (evaluated on the horizon) in powers of $\eta$ and taking the expectation value. Through order $\eta^{2}$ we get

$$
\begin{aligned}
\frac{d\left(M(v)+\langle\eta(v)\rangle_{\xi}\right)}{d v} & =-\left\langle\frac{B}{(M(v)+\eta(v))^{2}}\right\rangle_{\xi} \\
& =-\frac{B}{M^{2}(v)}\left[1-\frac{2}{M(v)}\langle\eta(v)\rangle_{\xi}+\frac{3}{M^{2}(v)}\left\langle\eta^{2}(v)\right\rangle_{\xi}+O\left(\frac{\eta^{3}}{M^{3}}\right)\right] .
\end{aligned}
$$

When the fluctuations become comparable to the mass itself, the third term (and higher order terms) on the righthand side is no longer negligible and we get non-trivial corrections to Eq. (8) for the dynamics of the mean value. These corrections can be interpreted as higher order radiative corrections to semiclassical gravity that include the effects of metric fluctuations on the evolution of the mean value. For instance, the third term on the right-hand side of Eq. (19) would correspond to a two-loop Feynman diagram involving a matter loop with an internal propagator for the metric perturbations (restricted to the spherically-symmetric sector in our case), as compared to just one matter loop, which is all that semiclassical gravity can account for.

An interesting aspect that we have not addressed in this work, but which is worth investigating, is the quantum coherence of those fluctuations. It seems likely that, given the long time periods involved and the size of the fluctuations, the entanglement between the Hawking radiation emitted and the black hole spacetime geometry will effectively decohere the large horizon fluctuations, rendering them equivalent to an incoherent statistical ensemble.

\footnotetext{
${ }^{7}$ Remember that for large black hole masses this can still correspond to physical distances much larger than the Planck length, as explained in Sec. III
} 
Does the existence of the significant deviations for the mean evolution mentioned above imply that the results based on semiclassical gravity obtained by Bardeen and Massar in Refs. 2, 3] are invalid? Several remarks are in order. First of all, those deviations start to become significant only after a period of the order of the evaporation time when the mass of the black hole has decreased substantially. Secondly, since fluctuations were not considered in those references, a direct comparison cannot be established. However, we can compare the average of the fluctuating ensemble with their results. Doing so exhibits an evolution that deviates significantly when the fluctuations become important. Nevertheless, if one considers a single member of the ensemble at that time, its evolution will be accurately described by the corresponding semiclassical gravity solution until the fluctuations around that particular solution become important again, after a period of the order of the evaporation time associated with the new initial value of the mass at that time.

In this paper we take a first step to put the study of metric fluctuations in black hole spacetimes on a firmer basis by considering a detailed derivation of the results from an appropriate formalism rather than using heuristic arguments or simple estimates. The spirit is somewhat analogous to the study of the mean back-reaction effect of Hawking radiation on a black hole spacetime geometry (both for black holes in equilibrium and for evaporating ones) by considering the solutions of semiclassical gravity in that case rather than just relying on simple energy conservation arguments. In order to obtain an explicit result from the stochastic gravity approach and compare with earlier work, in Sec. III we employed a simplifying assumption implicitly made in most of the literature: the existence of a simple connection between the outgoing energy flux fluctuations far from the horizon and the negative energy flux fluctuations crossing the horizon. In Sec. IV] we analyzed this assumption carefully and showed it to be invalid. This strongly suggests that one needs to study the stress tensor fluctuations from an explicit calculation of the noise kernel near the horizon. This quantity is obtainable from the stochastic gravity program and calculation is underway [43, 44].

A possible way to compute the noise kernel near the horizon could be to use an approximation scheme based on a quasilocal expansion such as Page's approximation [32] or similar methods corresponding to higher order WKB expansions [35]. ${ }^{8}$ With these techniques one can obtain an approximate expression for the Wightmann function of the matter fields, which is the essential object needed to compute the noise kernel. Unfortunately these approximations are only accurate for pairs of points with a small separation scale and break down when it becomes comparable to the black hole radius. Therefore, it cannot be employed to study the $l=0$ multipole since that corresponds to averaging the noise kernel over the whole solid angle, which involves typical separations for pairs of points on the horizon of the order of the black hole radius. Alternatively, one might hope to gain some insight on the fluctuations near a black hole horizon by studying the fluctuations of the event horizon surrounding any geodesic observer in de Sitter spacetime, which exhibits a number of similarities with the event horizon of a black hole in equilibrium [45]. In contrast to the black hole case, it may be possible to obtain exact analytical results for de Sitter space due to its high degree of symmetry.

Furthermore, as explained in Sec. [V] and shown in detail in Ref. [26], the noise kernel smeared over the horizon is divergent, and so are the induced metric fluctuations. Hence, one cannot study the fluctuations of the horizon as a three-dimensional hypersurface for each realization of the stochastic source because they are infinite, even when restricting one's attention to the $l=0$ sector. This means that one must find an adequate way of probing the metric fluctuations and extracting physically meaningful information, such as their effect on the Hawking radiation emitted by the black hole. One possibility is to study how metric fluctuations affect the propagation of a bundle of null geodesics [24, 25, 39, 46, 47]. One expects that this should provide a description of the effects on the propagation of a test field whenever the geometrical optics approximation is valid. However, if one tries to justify this point starting with a quantum field theory treatment, one realizes that even in simple cases interference effects cannot be neglected for sufficiently long times (much longer than the inverse of the frequency of the wave-packet whose propagation one is considering) and the geometrical optics approximation is invalid. Another possibility, which seems to constitute a better probe of the metric fluctuations, is to analyze the effect on the two-point quantum correlation functions of a test field. The two-point functions characterize the response of a particle detector for that field and can be used to obtain the expectation value and the fluctuations of the stress tensor of the test field.

Finally, since the large fluctuations suggested in Refs. 7, 8, 9, 10] involve time-scales much shorter than the evaporation time (contrary to those considered in this paper) and high multipoles, one expects that for a sufficiently massive black hole the spacetime near the horizon can be approximated by Rindler spacetime (identifying the black hole horizon and the Rindler horizon) provided that we restrict ourselves to sufficiently small angular scales. Thus, analyzing the effect of including the interaction with the metric fluctuations on the two-point functions of a test field propagating in flat space, which is technically much simpler, could provide useful information for the black hole case.

\footnotetext{
8 Note, however, that in most of these approaches the state of the quantum fields is the Hartle-Hawking vacuum. For an evaporating black hole, one should consider the Unruh vacuum.
} 


\section{Acknowledgments}

We thank Paul Anderson, Larry Ford, Valeri Frolov, Ted Jacobson, Don Marolf, Emil Mottola, Don Page, Renaud Parentani and Rafael Sorkin for useful discussions. This work is supported by NSF under Grant PHY03-00710.

[1] S. W. Hawking, Comm. Math. Phys. 43, 199 (1975).

[2] J. M. Bardeen, Phys. Rev. Lett. 46, 382 (1981).

[3] S. Massar, Phys. Rev. D 52, 5857 (1995).

[4] N. D. Birrell and P. C. W. Davies, Quantum fields in curved space (Cambridge University Press, Cambridge, 1994).

[5] R. M. Wald, Quantum field theory in curved spacetime and black hole thermodynamics (The University of Chicago Press, Chicago, 1994).

[6] E. E. Flanagan and R. M. Wald, Phys. Rev. D 54, 6233 (1996).

[7] R. D. Sorkin, in Proceedings of the Conference on Heat Kernel Techniques and Quantum Gravity, edited by S. A. Fulling (University of Texas Press, College Station, Texas, 1995), Discourses in Mathematics and its Applications, vol. 4, grqc/9508002.

[8] R. D. Sorkin, in Proceedings of the First Australasian Conference on General Relativity and Gravitation, edited by D. Wiltshire (University of Adelaide, Adelaide, Australia, 1997), gr-qc/9701056.

[9] A. Casher, F. Englert, N. Itzhaki, S. Massar, and R. Parentani, Nucl. Phys. B 484, 419 (1997).

[10] D. Marolf, in Particle physics and the universe, edited by J. Trampetic and J. Wess (Springer-Verlag, 2005), Springer Proceedings in Physics, vol. 98, hep-th/0312059.

[11] P. O. Mazur and E. Mottola, Proc. Nat. Acad. Sci. 111, 9545 (2004).

[12] C. H. Wu and L. H. Ford, Phys. Rev. D 60, 104013 (1999).

[13] B. L. Hu, A. Raval, and S. Sinha, in Black Holes, Gravitational Radiation and the Universe: Essays in honor of C. V. Vishveshwara, edited by B. Iyer and B. Bhawal (Kluwer Academic Publishers, Dordrecht, 1998), gr-qc/9901010.

[14] S. Sinha, A. Raval, and B. L. Hu, Found. Phys. 33, 37 (2003).

[15] J. D. Bekenstein, in Quantum Theory of Gravity, edited by S. M. Christensen (Adam Hilger, Bristol, 1984).

[16] C. P. Burgess, Living Rev. Rel. 7, 5 (2004).

[17] J. F. Donoghue, Phys. Rev. D 50, 3874 (1994).

[18] J. F. Donoghue, in The Eighth Marcel Grossmann Meeting, edited by T. Piran and R. Ruffini (World Scientific, Singapore, 1999), gr-qc/9712070.

[19] S. Weinberg, hep-th/0506236.

[20] B. L. Hu and E. Verdaguer, Class. Quant. Grav. 20, R1 (2003).

[21] B. L. Hu and E. Verdaguer, Living Rev. Rel. 7, 3 (2004).

$[22]$ A. Roura and E. Verdaguer, in preparation.

[23] B. L. Hu, A. Roura, and E. Verdaguer, Phys. Rev. D 70, 044002 (2004).

[24] R. Parentani, Int. J. Theor. Phys. 40, 2201 (2001).

[25] R. Parentani, Int. J. Theor. Phys. 41, 2175 (2002).

[26] B. L. Hu and A. Roura, in preparation.

[27] C. W. Misner, K. S. Thorne, and J. A. Wheeler, Gravitation (Freeman, San Francisco, 1973).

[28] R. M. Wald, General Relativity (The University of Chicago Press, Chicago, 1984).

[29] D. N. Page, Phys. Rev. D 13, 198 (1976).

[30] J. W. York, Phys. Rev. D 31, 775 (1985).

[31] P. Candelas, Phys. Rev. D 21, 2185 (1980).

[32] D. N. Page, Phys. Rev. D 25, 1499 (1982).

[33] K. W. Howard and P. Candelas, Phys. Rev. Lett. 53, 403 (1984).

[34] K. W. Howard, Phys. Rev. D 30, 2532 (1984).

[35] P. R. Anderson, W. A. Hiscock, and D. A. Samuel, Phys. Rev. D 51, 4337 (1995).

[36] S. P. Trivedi, Phys. Rev. D 47, 4233 (1993).

[37] A. Strominger and S. P. Trivedi, Phys. Rev. D 48, 5778 (1993).

[38] F. C. Lombardo, F. D. Mazzitelli, and J. G. Russo, Phys. Rev. D 59, 064007 (1999).

[39] R. Parentani, Phys. Rev. D 63, 041503 (2001).

[40] A. Campos and B. L. Hu, Phys. Rev. D 58, 125021 (1998).

[41] A. Campos and B. L. Hu, Int. J. Theor. Phys. 38, 1253 (1999).

[42] L. H. Ford and T. A. Roman, Phys. Rev. D 72, 105010 (2005).

[43] N. G. Phillips and B. L. Hu, Phys. Rev. D 63, 104001 (2001).

[44] N. G. Phillips and B. L. Hu, Phys. Rev. D 67, 104002 (2003).

[45] G. W. Gibbons and S. W. Hawking, Phys. Rev. D 15, 2738 (1977).

[46] C. Barrabès, V. Frolov, and R. Parentani, Phys. Rev. D 59, 124010 (1999).

[47] C. Barrabès, V. Frolov, and R. Parentani, Phys. Rev. D 62, 044020 (2000). 